\title{
Proceedings
}

\section{Designing a Rogowski Coil with Particle Swarm Optimization ${ }^{+}$}

\author{
Guillermo Robles ${ }^{1, *(1)}$, Muhammad Shafiq ${ }^{2}$ al and Juan Manuel Martínez-Tarifa ${ }^{1}(\mathbb{C}$ \\ 1 Department of Electrical Engineering, Universidad Carlos III de Madrid, Avda, Universidad, \\ 30, Leganés, 28911 Madrid, Spain; grobles@ing.uc3m.es; jmmtarif@ing.uc3m.es \\ 2 School of Technology and Innovations, Electrical Engineering, University of Vaasa, Wolffintie 34, \\ 65200 Vaasa, Finland; muhammad.shafiq@uwasa.fi \\ * Correspondence: grobles@ing.uc3m.es \\ + Presented at the 5th International Electronic Conference on Sensors and Applications, 15-30 November 2018; \\ Available online: https:/ / ecsa-5.sciforum.net.
}

Published: 14 November 2018

\begin{abstract}
Rogowski coils are inductive sensors based on Faraday's and Ampère's Laws to measure currents through conductors without galvanic contact. The main advantage of Rogowski coils when compared with current transformers is the fact that the core is air so they never saturate and the upper cut-off current can be higher. These characteristics make Rogowski coils ideal candidates to measure high-amplitude pulsed currents. However, there are two main drawbacks. On the one hand, the output voltage is the derivative of the primary current so it has to be integrated to measure the original signal; on the other hand, the transfer function is resonant as a result of the capacitance and the self-inductance of the coil. The solution is the use of a passive integration with a terminating resistor at the output of the sensor that splits the two complex poles and gives a constant transfer function for a determined bandwidth. The downside is a loss of sensitivity. Since it is possible to calculate the electrical parameters of the coil based on its geometrical dimensions, the geometry can be adapted to design sensors for different applications depending on the time characteristics of the input current. This paper proposes the design of Rogowski coils based on their geometric characteristics maximizing the gain-bandwidth product using particle swarm optimization and adapting the coils to the specific requirements of the application.
\end{abstract}

Keywords: Rogowski coils; particle swarm optimization; gain-bandwidth product; current measurement; magnetic field measurement

\section{Introduction}

Among high-frequency current measurement sensors, current transformers with ferrite cores and Rogowski coils are the preferred solution particularly because of their non-intrusive measurement capability [1]. However, on the basis of critical parameters, such as cost, bandwidth, sensitivity, saturation, linearity, operating temperature, integrability, flexibility and isolation, and material technology, Rogowski coils have been considered as the favorite tool in industrial applications [2]. Nowadays, Rogowski coils are being used in various measurement applications. These not only include normal operations but also fault situations such as AC and transient measurements, partial discharges, ground fault situations, and relay protection tasks [2]. This is because of their capability of measuring signals with frequencies ranging from a few hertz up to several megahertz and with an amplitude of few amperes to above a kiloampere. The physical layout of the coil, signal processing, and signal integration are the important issues in the design of these types of sensors [3]. A lot of work has been published on the design and analysis of Rogowski coils and still there are various aspects that need to be explored further to improve their performance [1]. 
The selection of suitable geometrical parameters has been identified as an important aspect regarding the proper operation and installation of the coil around the power component being tested $[4,5]$. These parameters determine the electrical parameters and significantly affect the measuring performance of the coil in terms of its sensitivity and bandwidth. A higher sensitivity and wider bandwidth are generally desired in the design of the Rogowski coil sensor; however, changing one geometrical parameter may negatively affect the sensitivity and bandwidth, for example, an increase in sensitivity may decrease the bandwidth and vice versa [4]. Therefore, it is quite challenging to develop a Rogowski coil design that maximizes the sensitivity and bandwidth of the coil simultaneously for current measurement based on the arbitrary selection of the geometric factors. In this paper, a formulated method is presented to determine the optimized geometrical parameters based on the Particle Swarm Optimization (PSO) technique in order to have the maximum sensitivity for a certain bandwidth.

\section{Rogowski Coil Geometry and Electrical Parameters}

The behavior of a Rogowski coil in terms of sensitivity and bandwidth is determined by its electrical parameters: resistance $R$, self-inductance $L$, capacitance between the winding and the returning turn $C_{l}$, and capacitance between turns $C_{t}$. In most cases, $C_{t}$ is negligible compared with $C_{l}$, so henceforth, we will only consider $C_{l}$. Finally, $M$ is the mutual inductance between the primary conductor and the coil. These parameters can be expressed in function of the geometry of the coil with the following equations [6]:

$$
\begin{gathered}
R=\rho \frac{4 l}{\pi d^{2}} \\
L=\frac{\mu_{0}}{2 \pi} N^{2} h \log \frac{b}{a} \\
C_{l}=\frac{\pi^{2} \epsilon_{0}(a+b)}{2 \sqrt{A / \pi}} \frac{2 \sqrt{d}}{\log \frac{b}{a}} \\
M=\frac{\mu_{0}}{2 \pi} N h \log \frac{b}{a}
\end{gathered}
$$

where, according to Figure $1, a$ is the internal diameter of the coil, $b$ is the external diameter, $h$ is the height of the coil, $N$ is the number of turns, and $A=(b-a) h / 2$ is the area of a turn. Additionally, $d$ and $l$ are the diameter and the total length of the wire, respectively; $\rho, \mu_{0}$, and $\epsilon_{0}$ are the resistivity of copper, the magnetic permeability, and the permittivity of air, respectively.

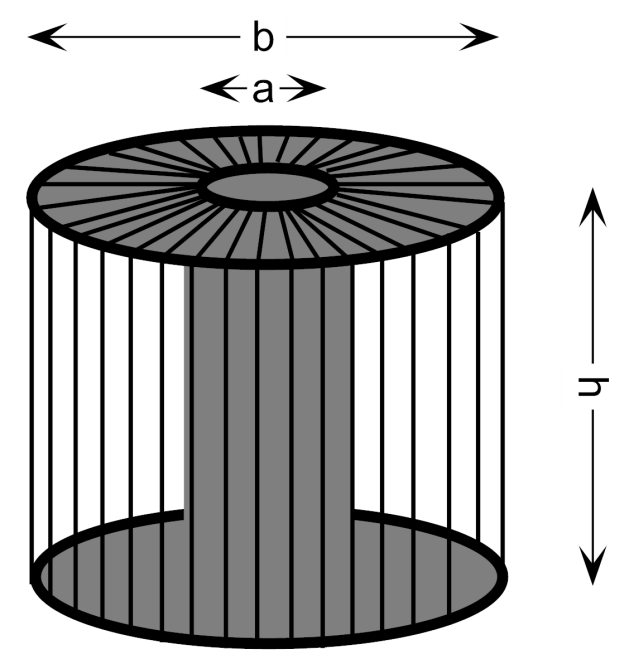

Figure 1. Geometrical parameters of a Rogowski coil. 


\section{Frequency Response of a Rogowski Coil}

Considering the equivalent circuit in lumped parameters shown in Figure 2, the output of the Rogowski coil in volts can be obtained with the transfer function shown in (5). Using the electrical parameters from a Rogowski coil manufactured and characterized in Aalto University, $L=1.2 \mu \mathrm{H}$, $C_{l}=5.7 \mathrm{pF}, M=9.51 \mu \mathrm{H}$ and considering $R=0.1 \Omega$, the resulting Bode plot is represented in Figure 3 . The resistance of the selected Rogowski coil is actually $R=0.005 \Omega$ in direct current for $N=30$ and an AWG 18 wire. However, the bandwidth of these types of sensors can reach tens of megahertz so it was decided to multiply the resistance 20 times to take into account the skin effect, a value similar to those obtained from previous works in this frequency range. Nevertheless, this resistance can be neglected in most cases and does not contribute to the frequency response notably. It can be seen in the Bode plot that the output voltage $v(t)$ is the derivative of the input $i(t)$ (when the phase is $+90^{\circ}$ ) up to frequencies close to the resonance frequency in $f_{0}=1 / 2 \pi \sqrt{L C_{l}}=60.8 \mathrm{MHz}$.

$$
V_{o}(s)=\frac{M s}{L C_{l} s^{2}+R C_{l} s+1} I(s)
$$

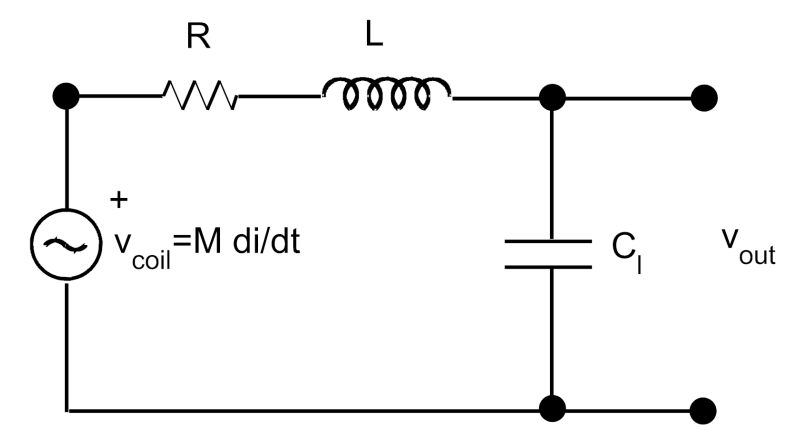

Figure 2. Electrical model of a Rogowski coil considering lumped parameters.

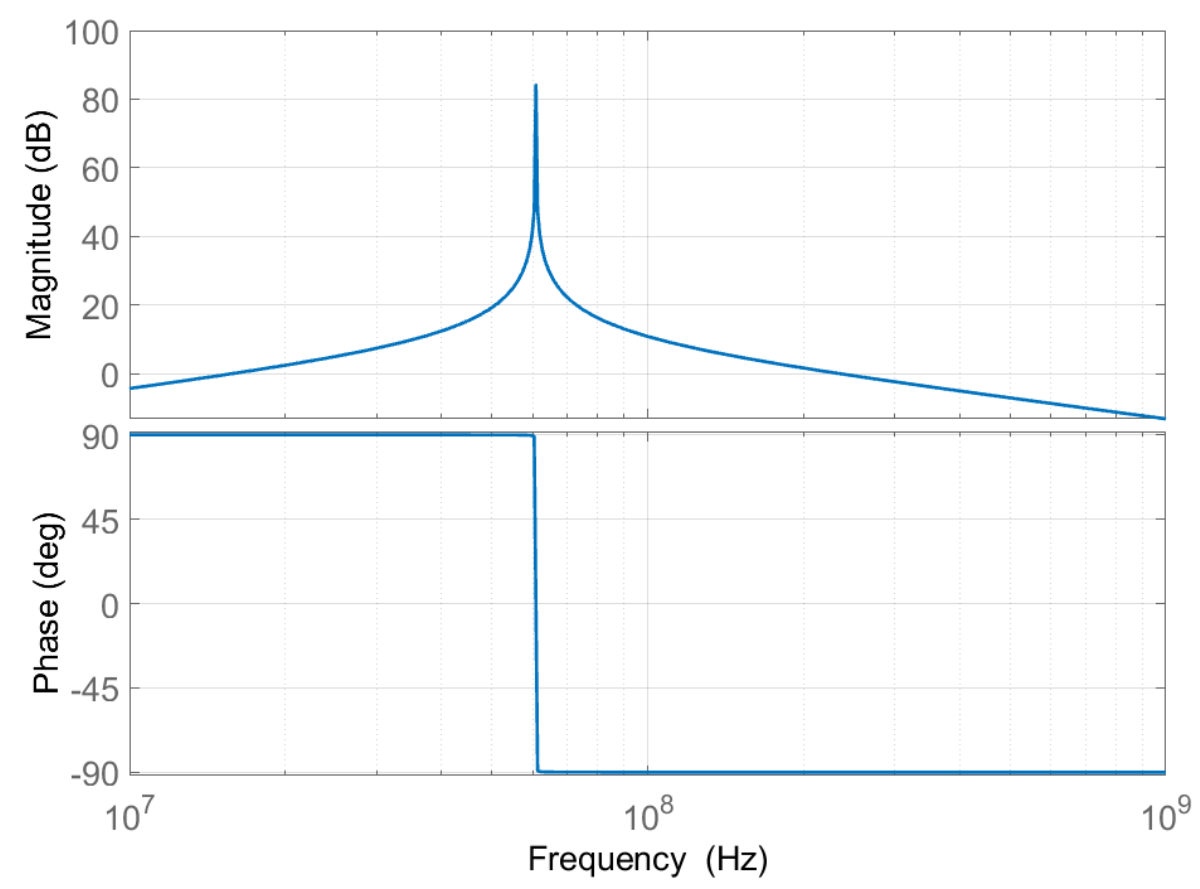

Figure 3. Frequency response of an underdamped second order system corresponding to a Rogowski coil. 
There is the possibility of obtaining an output voltage proportional to the input voltage using a resistor at the ends of the Rogowski coil that will split the complex pole into two poles [3]. Consider the transfer function in Equation (6) which includes a generic terminating resistance $R_{t}=10 \Omega$ in parallel with the capacitance in Figure 2. Now, there is a band of frequencies between 10 and $100 \mathrm{MHz}$ where the input and the output are in phase so the output is proportional to the input, see the graph in Figure 4. Unfortunately, the sensitivity of the coil drops to close to $-30 \mathrm{~dB}$.

$$
V_{o}(s)=\frac{R_{t} M s}{L R_{t} C_{l} s^{2}+\left(L+R R_{t} C_{l}\right) s+R+R_{t}} I(s)
$$

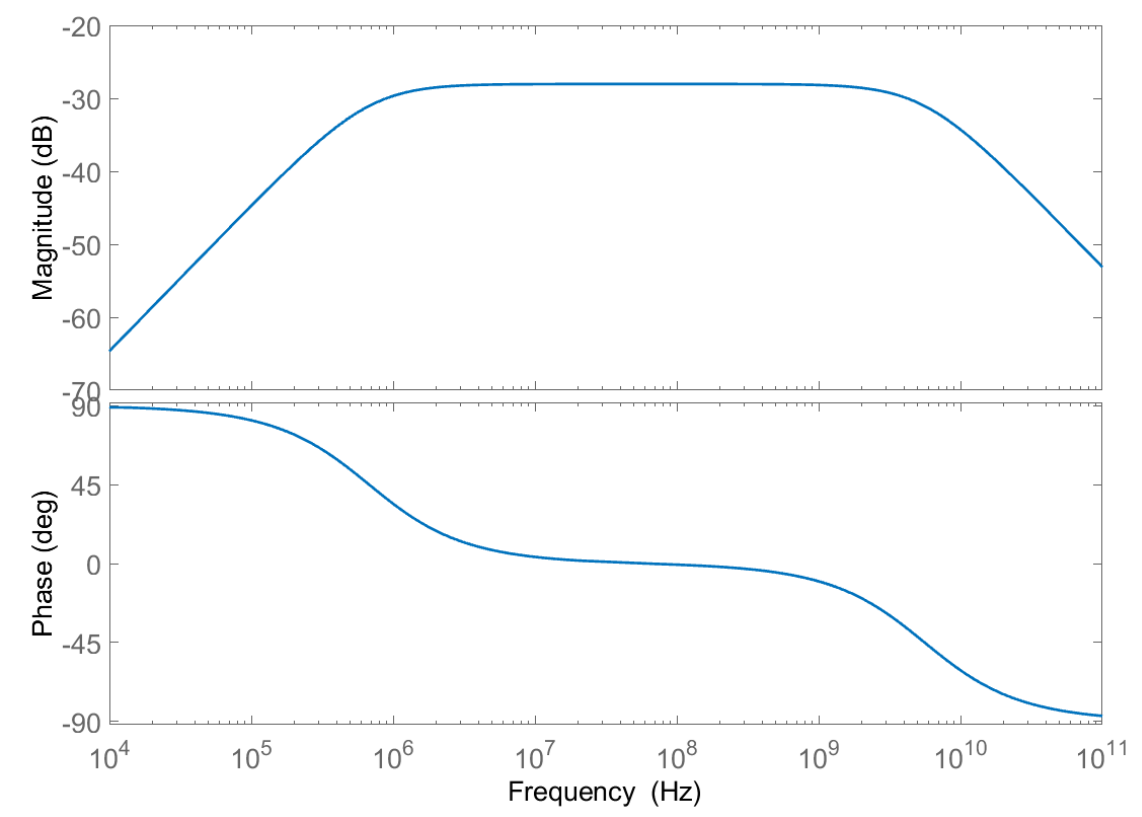

Figure 4. Frequency response of a Rogowski coil with terminating resistance.

\section{Optimizing the Geometry of a Rogowski Coil}

The objective now is the design of a self-integrating Rogowski coil optimizing the gain-bandwidth product (GBWP) to minimize the loss of sensitivity while maximizing the bandwidth of the coil. The optimization function will be based on the geometrical parameters, and hence, the electrical parameters will also be determined. Then, the design variables will be $a, b, h, N$, and $R_{t}$, the last one being the most important to select the gain of the Rogowski coil . There are also some spatial constraints: the internal diameter should be large enough to allow the passing of the primary conductor so it was set to a minimum of $10 \mathrm{~cm}$. The external diameter should be larger than $a$ and with an upper bound set to $30 \mathrm{~cm}$ so the coil does not interfere with the close instrumentation. The height of the coil has to be selected between 1 and $4 \mathrm{~cm}$ for the same reasons. Additionally, the minimum number of turns is $N=3$ and the minimum terminating resistance $R_{t}=10 \Omega$. Finally, the bandwidth must be chosen between $150 \mathrm{kHz}$ and $150 \mathrm{MHz}$ to be able to measure currents in the HF-VHF ranges. Notice that since the transfer function is second order, the effective band of frequencies where the input and output are in phase would be very narrow.

The optimization method is based on Particle Swarm Optimization in a five-dimensional space corresponding to the five design parameters. This method places a flock or swarm of particles randomly in the solution space [7]. In every iteration $l$, every particle evaluates the GBWP in its position determined by the design parameters. The best solution for the GBWP of each particle $k$, $\mathbf{P}_{\mathbf{k}, \text { best }}(l)$ is registered and updated in every iteration, as well as the best global solution of all the 
swarm, $\mathbf{P}_{\text {best }}(l)$. These two possible solutions are part of the swarm intelligence so every particle knows its own best fit and the particle with the best approximation to the solution. Then, the positions of the particles are changed considering the velocity of particle $k$ that ties it to its best position and another component that moves the particle towards the global best [8], according to Equations (7).

$$
\begin{aligned}
\mathbf{v}_{\mathbf{k}}(l+1) & =\mathbf{v}_{\mathbf{k}}(l)+\mathbf{U}_{\mathbf{1}}(0,1) \otimes\left[\mathbf{P}_{\mathbf{k}, \text { best }}(l)-\mathbf{P}_{\mathbf{k}}(l)\right] \\
& +\mathbf{U}_{\mathbf{2}}(0,1) \otimes\left[\mathbf{P}_{\text {best }}(l)-\mathbf{P}_{\mathbf{k}}(l)\right], \\
\mathbf{P}_{\mathbf{k}}(l+1) & =\mathbf{P}_{\mathbf{k}}(l)+\mathbf{v}_{\mathbf{k}}(l+1) .
\end{aligned}
$$

with $\mathbf{U}_{1}(0,1)$ and $\mathbf{U}_{\mathbf{2}}(0,1)$ being five dimensional line matrices whose elements are uniformly distributed between 0 and 1 .

After running the algorithm with a swarm of 2000 particles, the resulting parameters for the optimized Rogowski coil are presented in Table 1. The geometrical parameters led to higher values of $b$ and $h$ than those from the coil in Figure 4, as suggested by previous studies [6]. In addition, the use of higher values of $R_{t}$ are consistent with an increase in the gain.

Table 1. Parameters of the Rogowski coil optimizing the gain-bandwidth product (GBWP).

\begin{tabular}{cc}
\hline Parameter & Value \\
\hline $\mathrm{a}$ & $10 \mathrm{~cm}$ \\
$\mathrm{~b}$ & $19.9 \mathrm{~cm}$ \\
$\mathrm{~h}$ & $4 \mathrm{~cm}$ \\
$\mathrm{~N}$ & $205 \mathrm{turns}$ \\
$R_{t}$ & $226.5 \Omega$ \\
\hline
\end{tabular}

The resulting Bode plot for the designed coil is represented in Figure 5. It can be seen that the bandwidth goes from $810 \mathrm{kHz}$ to $2.4 \mathrm{MHz}$ as long as the phase shift introduced by the transfer function is sufficiently close to $0^{\circ}$, and provided it is in the interval between $10^{\circ}$ and $-10^{\circ}$. The gain is close to $0 \mathrm{~dB}$ (which also represents the widest bandwidth), much larger than in the case presented in Figure 4 .

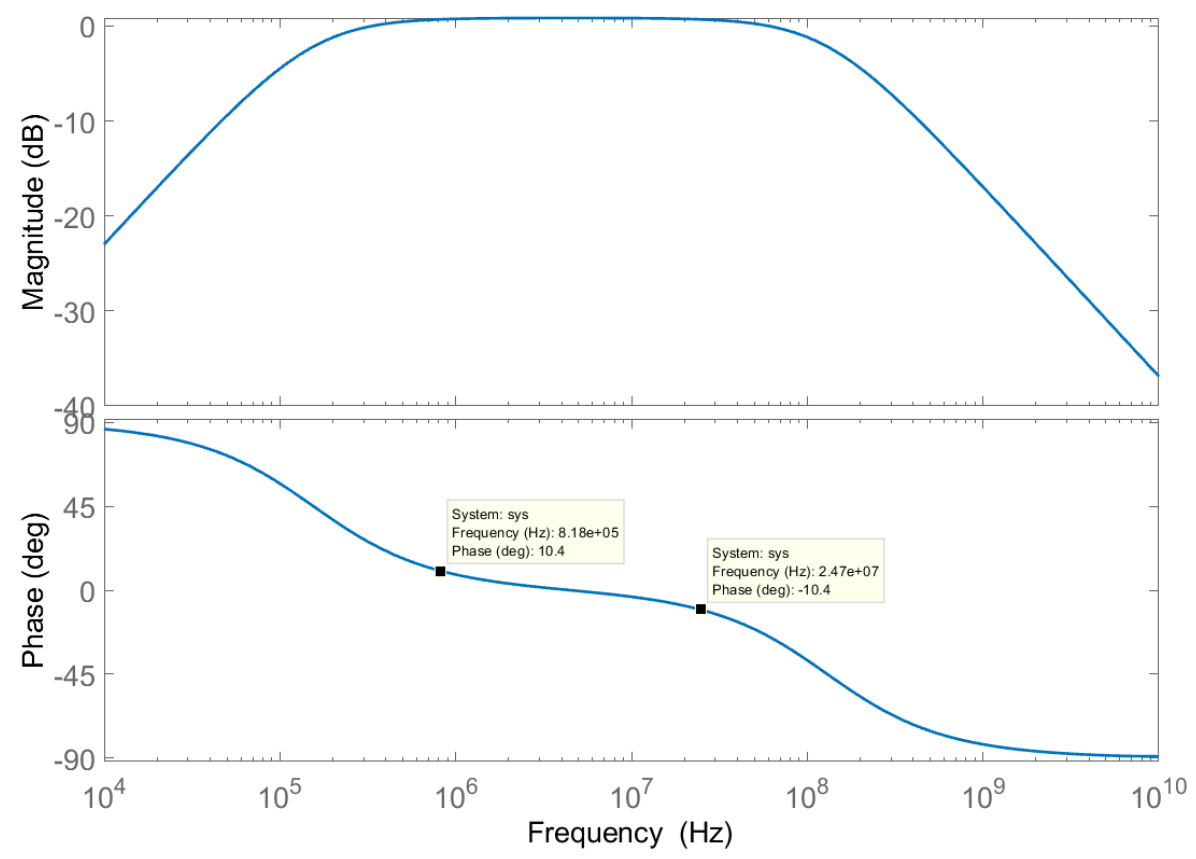

Figure 5. Frequency response of the designed Rogowski coil. 


\section{Discussion}

This work proposes a flexible method to design a Rogowski coil with optimum gain-bandwidth parameters. The equations which relate the geometrical characteristics with the electrical properties of a Rogowski coil are based on the assumption that the values of resistance, inductance, and capacitance are not distributed along the wire. This is true if the length of the wire is short compared with the wavelength of the voltage and current in the coil. As regards the designed coil, the length of a turn is $0.18 \mathrm{~m}$ and the total length of the wire is $36.9 \mathrm{~m}$ plus the length of the returning coil. The wavelength of signals with a frequency of $1 \mathrm{MHz}$ traveling at the speed of light is $300 \mathrm{~m}$ so the assumption regarding the lumped model would be consistent with these. Nevertheless, the design seems effective considering it is a distributed model, so the next step would be to construct the coil and test its frequency response especially focusing on signals near to the high cut-off frequency of $2.4 \mathrm{MHz}$.

In any case, the method presented is notably flexible, therefore, it can be applied to other geometries and constraints thus optimizing any type of Rogowski coil.

Author Contributions: The idea of the paper and the implementation of the algorithm was done by G.R. A thorough review of the state of the art and the manufacturing of the generic Rogowski coil at Aalto University was made by M.S. The analysis of the results was made by G.R. and J.M.M-T. The writing, review and editing of the paper was done by all the authors.

Funding: This research has received funds from the Academy of Finland under the Grant No. 309412 and the Spanish Government (MINECO) and the European Regional Development Fund (ERDF) under contract DPI2015-66478-C2-1-R (MINECO/FEDER, UE).

Conflicts of Interest: The authors declare no conflict of interest.

\section{References}

1. Samimi, M.H.; Mahari, A.; Farahnakian, M.A.; Mohseni, H. The Rogowski Coil Principles and Applications: A Review. IEEE Sens. J. 2015, 15, 651-658.

2. Hu, C.; Li, H.B.; Jiao, Y.; Zhang, Z.; Li, K. Comparative analysis of various models of Rogowski coil for very fast transient study. IEEJ Trans. Electr. Electron. Eng. 2018, 13, 1319-1327.

3. Argüeso, M.; Robles, G.; Sanz, J. Implementation of a Rogowski coil for the measurement of partial discharges. Rev. Sci. Instrum. 2005, 76, 065107.

4. Shafiq, M.; Hussain, G.A.; Kütt, L.; Lehtonen, M. Effect of geometrical parameters on high frequency performance of Rogowski coil for partial discharge measurements. Measurement 2014, 49, 126-137.

5. Liu, Y.; Lin, F.; Zhang, Q.; Zhong, H. Design and Construction of a Rogowski Coil for Measuring Wide Pulsed Current. IEEE Sens. J. 2011, 11, 123-130.

6. Robles, G.; Martinez, J.M.; Sanz, J.; Tellini, B.; Zappacosta, C.; Rojas, M. Designing and tuning an air-cored current transformer for partial discharges pulses measurements. In Proceedings of the 2008 IEEE Instrumentation and Measurement Technology Conference, Victoria, BC, Canada, 12-15 May 2008; pp. 2021-2025.

7. Kennedy, J.; Eberhart, R. Particle swarm optimization. In Proceedings of the ICNN'95-International Conference on Neural Networks, Perth, WA, Australia 27 November-1 December 1995; Volume 4, pp. 1942-1948.

8. Poli, R.; Kennedy, J.; Blackwell, T. Particle swarm optimization. Swarm Intell. 2007, 1, 33-57. 